\title{
Structure formation by cosmic strings with a cosmological constant
}

\author{
Richard A. Battye ${ }^{1}$, James Robinson ${ }^{2}$ and Andreas Albrecht ${ }^{3}$ \\ ${ }^{1}$ Department of Applied Mathematics and Theoretical Physics, University of Cambridge, \\ Silver Street, Cambridge CB3 9EW, U.K. \\ ${ }^{2}$ Department of Astronomy, Campbell Hall, University of California, Berkeley CA 94720, U.S.A. \\ ${ }^{3}$ Theoretical Physics Group, Blackett Laboratory, Imperial College, Prince Consort Road, London SW7 2BZ, U.K.
}

\begin{abstract}
We investigate cosmic string models for structure formation which include a non-zero cosmological constant. We find that the background evolution of density perturbations and modifications to the scaling behaviour of the strings both act to increase the amount of power present on $100 h^{-1} \mathrm{Mpc}$ scales. We estimate the size of this effect using an analytic model for the evolution of the string network and find that a bias $b \sim 2$ can give acceptable agreement with the current observations. An interesting by product of these modifications is a broad peak in the cosmic microwave background (CMB) angular power spectrum around $l=400-600$.
\end{abstract}

PACS Numbers : $98.80 . C q, 95.35+d$

Topological defect theories, such as cosmic strings [1], have for many years represented the only serious alternatives to the standard inflationary paradigm as theories for structure formation. In inflationary theories, structure in the universe is seeded by causal processes operating outside the framework of the Standard Big Bang (SBB) cosmology, which imprint a primordial spectrum of adiabatic density perturbations on all scales. By contrast, in defect theories the universe is taken to be initially smooth and perturbations must be seeded by the causal action of an evolving network of defect seeds, within the background of the SSB cosmology.

Recent work 24 has pointed to a serious problem for defect theories reconciling the amplitude of large scale $\mathrm{CMB}$ anisotropies with that in the matter distribution on scales around $100 h^{-1} \mathrm{Mpc}$, in a flat universe where the matter density is critical, that is $\Omega_{m}=1$. In refs. [3, 4, this problem was quantified in terms of $b_{100}$, where $b_{R}$ is the bias between the cold dark matter (CDM) and the galaxy distribution in a sphere of radius $R h^{-1} \mathrm{Mpc}$ that we must postulate in order to make a given theory consistent with the data. It was argued that unacceptably large biases, $b_{100} \simeq 5$, would be required in order to make scaling defect theories with $\Omega_{m}=1$ compatible with current observations. We named this the $b_{100}$ problem and suggested that its resolution is the most serious challenge facing defect models for structure formation.

These recent calculations (see also ref. 50) represent the first calculations using a linear Einstein-Boltzmann solver which includes all the relevant physical effects. The only input required is the unequal time-correlator (UETC) for the defect stress-energy, or an ensemble of source histories with the same UETC. In refs. [3, 4] we used a sophisticated model to represent the stress-energy

\footnotetext{
${ }^{1}$ The present calculations and those of refs. 1 , used a modified version CMBFAST [6] which has been shown to give accurate $(\sim 1 \%)$ results for most inflationary models.
}

of a network of cosmic strings, while numerical simulations were used in refs. 2, 5].

In the past, uncertainties in the results of defect calculations have meant that work has tended to concentrate on the simplest possible models. Some attempts have been made to modify the semi-analytic approach of Albrecht and Stebbins [7] to include additional parameters, such as curvature (an open universe, $\Omega_{k}$ ) or a cosmological constant $\left(\Omega_{\Lambda}\right)$ [8,9]. This method yields only the shape of the matter power spectrum, although in ref. [9] a simple model was employed to estimate the dependence of the large angle fluctuation amplitude on $\Omega_{\Lambda}$ and $\Omega_{k}$, allowing rough statements about the relative $\mathrm{CMB} /$ matter normalization to be made. The results of these calculations - reduced bias required to reconcile theory with the data, and an improvement to the shape of the matter spectrum — serve as impetus for the current work, which considers a flat universe with a non-vanishing cosmological constant. Our calculations use the same modified version of CMBFAST and source two-point functions as in refs. [3, 14] and represent a self consistent calculation of both the CDM and CMB power spectra over all observable scales. We should note that similar results for the matter power spectra have been found by Avelino et al [10].

The introduction of a cosmological constant will be seen to improve the required value of the bias in two ways. The first is in terms of the modified evolution of the background spacetime, which is illustrated in Fig. 1. This shows the results for our standard scaling string source (described in detail in [4]) with various choices of the parameter $\Omega_{\Lambda}$, the proportion of the critical density from the cosmological constant with the Hubble constant $H_{0}=100 h \mathrm{~km} \mathrm{sec}^{-1} \mathrm{Mpc}^{-1}$ given by $h=0.5$. We find that as $\Omega_{\Lambda}$ is increased, the amount of power on large scales (around $100 h^{-1} \mathrm{Mpc}$ ) is increased, with a corresponding decrease on small scales, bringing the shape of the matter spectrum in line with the galaxy data and reducing the magnitude of the $b_{100}$ problem. This modification can be understood via the standard shape pa- 


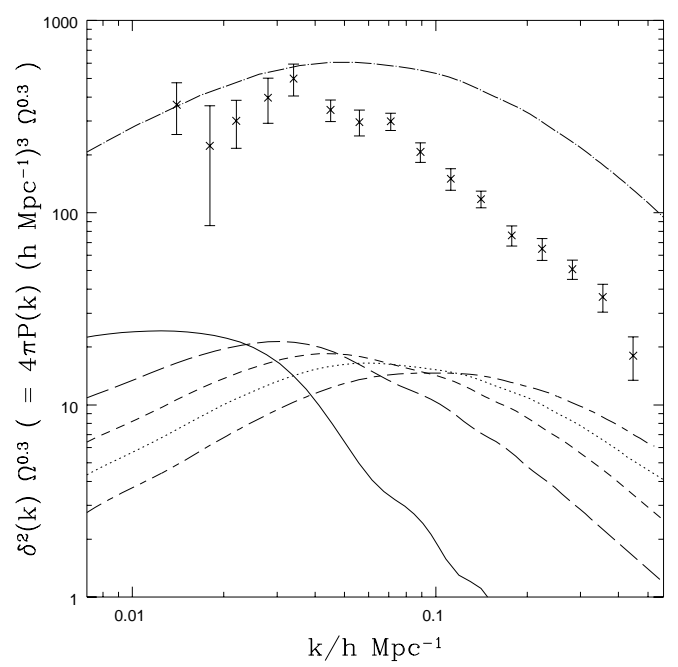

FIG. 1. The (COBE normalized) matter power from the scaling cosmic string model for various values $\Omega_{\Lambda} . \Omega_{\Lambda}=0.0$ (short-long dashed line), 0.3 (dotted dashed line), 0.5 (short dashed line), 0.7 (long dashed line) and 0.9 (solid line). Included also are the observed data points 11] and the Standard Cold Dark Matter model (dot-dashed curve).

rameter $\Gamma \approx \Omega_{m} h$ and the equivalent shift in the time of radiation-matter equality. We find that $\Omega_{\Lambda}=0.7$ seems to reproduce the shape of the observed spectrum particularly well, although assuming perfect scaling this still requires a bias $b \approx 4$, over all scales. Note that we plot $P(k) \Omega_{m}^{0.3}$ rather than just $P(k)$, so as to undo the redshift space distortion calculated in ref. [11].

In our previous work [3 3 ] we have made an extensive study of the effects of deviations from scaling. In the case of $\Omega_{\Lambda}=0.0$, we found that only very radical deviations from scaling can improve the amount of power on $100 h^{-1} \mathrm{Mpc}$ scales, at the expense of creating an over production of power on smaller scales. However, given the improvement in the shape and amplitude of the matter power spectrum from the inclusion of a cosmological constant, one might think that mild deviations from scaling might improve things further.

Rather than using the arbitrary scaling deviations to deduce a transition which yields the best case scenario, for the bulk of this work we have used the velocity dependent one-scale model for string evolution [12], which was also used as the basis for the string evolution in ref. [9]. This allows us to calculate the three quantities which are variables in our model, namely the string coherence length relative to the horizon $\xi(\tau)$, the rms string velocity $v(\tau)$ and the string density $\rho_{s}(\tau)=\mu / \xi^{2}$, as functions of conformal time, where $\mu$ is the mass per unit length of the string. The evolution equations for this model become

$$
\ell^{\prime}=\mathcal{H} \ell v^{2}+\frac{1}{2} \tilde{c} v, \quad v^{\prime}=\left(1-v^{2}\right)\left(\frac{\tilde{k}}{\ell}-2 \mathcal{H} v\right)
$$

where $\ell$ is the length scale on the network, $\mathcal{H}=a^{\prime} / a, \tilde{c}(\tau)$

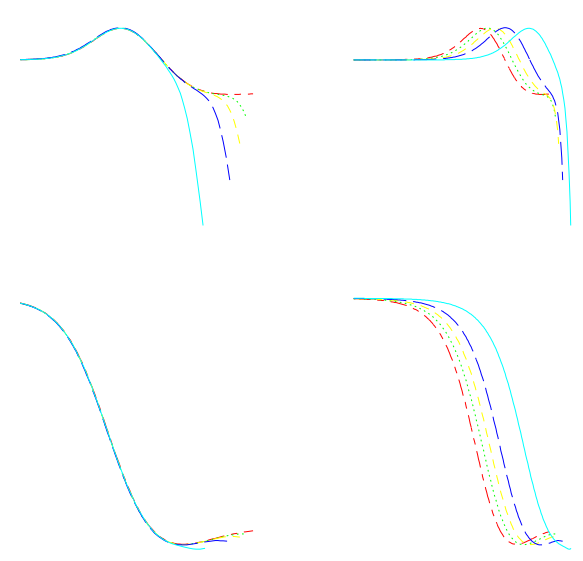

FIG. 2. The results of the velocity dependent one-scale model for the same values of $\Omega_{\Lambda}$ as in Fig. 1. On left the density of strings relative to the background against $a / a_{\text {eq }}$ (bottom) and the rms velocity of the strin gs (top). On the right the same quantities but this time against $\tau$.

is the chopping efficiency and $\tilde{k}(\tau)$ is the curvature of the strings. In order to interpolate between the measured values in the radiation and matter dominated eras, we use

$$
\tilde{c}(\tau)=\frac{c_{\mathrm{r}}+a(\tau) g c_{\mathrm{m}}}{1+a(\tau) g}, \quad \tilde{k}(\tau)=\frac{k_{\mathrm{r}}+a(\tau) g k_{\mathrm{m}}}{1+a(\tau) g},
$$

where $c_{\mathrm{r}}=0.23, k_{\mathrm{r}}=0.17$ and $c_{\mathrm{m}}=0.18, k_{\mathrm{m}}=0.49$, the values in the radiation and matter eras respectively, and $g=0.19$ is a constant which determines the speed of the transition. It has been suggested [13] that this model reproduces the behaviour of a string network through the radiation-matter transition.

The solution of these equations is illustrated in Fig. 2 for various values of $\Omega_{\Lambda}$ and $h=0.5$. We see that the density of strings shifts by approximately a factor of two in the interval $a / a_{\mathrm{eq}}=1$ to 100 independent of the value of $\Omega_{\Lambda}$ and in the models with $\Omega_{\Lambda} \neq 0$ there is a further shift in the string density, during the $\Lambda$ dominated phase; the effect being most obvious for large $\Omega_{\Lambda}$. But when plotted against $\tau$, we see that $\tau_{\text {eq }}$, and hence the transition era, is shifted as $\Omega_{m}^{-1}$ in conformal time. For $\Omega_{m}=1$, the velocity interpolates between the measured values in the radiation and matter eras, with a transient increase during the transition, and for $\Omega_{\Lambda} \neq 0$, the velocity of the strings is exponentially suppressed during the $\Lambda$ dominated era. Physically, the cosmological constant is causing the universe to expand exponentially, reducing the velocity of the strings, preventing reconnection and hence the strings are no longer scaling.

Here, we comment briefly on the implementation of these deviations from scaling into our string model. The velocity and length of each string segment at each time 


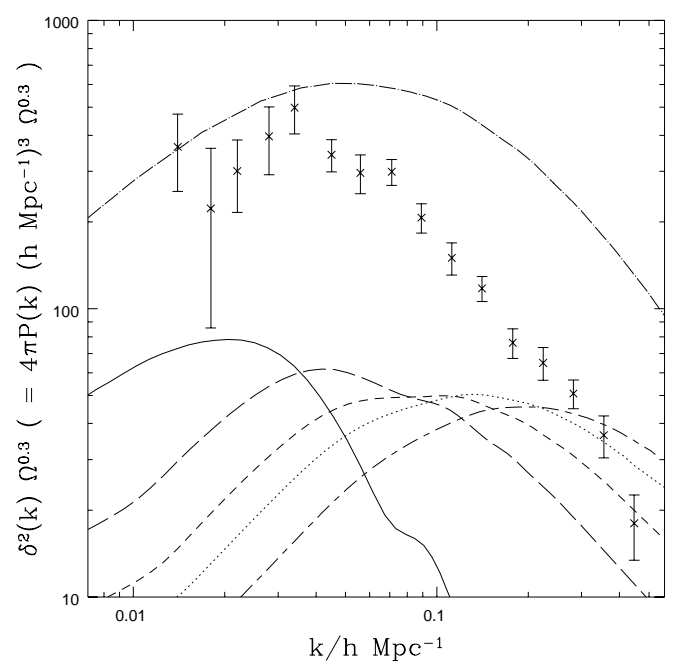

FIG. 3. The (COBE) normalized matter power spectrum for the same values of $\Omega_{\Lambda}$ as in Fig. 1, but this time using the velocity dependent one scale model to calculate $\xi$ and $v$. Once again we include the observed data point s and Standard CDM.

are simply chosen to be $v(\tau)$ and $\xi(\tau)$, but for technical reasons we now pick a fixed value of $v$ rather than choosing it from a random distribution, which has been shown to make very little difference to the resulting power spectrum. The number density of strings is taken to be $(\xi(\tau) \tau)^{-3}$, that is, there is one string per correlation length cubed.

Figs. 3 and 1 show the matter power spectra and $\mathrm{CMB}$ anisotropies for the same values of $\Omega_{\Lambda}$ as Fig. 1, with $\xi$, $v$ and number density of strings given by this model. Coupled with the effects of the background, the deviations from scaling improve $b_{100}$ significantly for $\Omega_{\Lambda} \neq 0$, with the best fit for $\Omega_{\Lambda}=0.7$ (and hence, for a similar value of $\Omega_{m}$ as favoured in refs. [8] (-9]), although the inclusion of the time dependence on $\xi$ has slightly affected the shape of the power spectrum. We estimate that a bias of $b_{100}=2.6$ would give a good fit to the observations on large scales at the expense of an overproduction of power on smaller scales. A robust feature of the CMB spectra appears to be a peak at around $l=400-600$, whose height is increased as $\Omega_{\Lambda}$ increases. Finally, we should note that the value of string density per unit length $G \mu$ required to normalize to COBE is around $1 \times 10^{-6}$, almost independent of $\Omega_{\Lambda}$, if we assume that the effective mass per unit length is $\tilde{\mu} \approx 1.7 \mu$. This is well below the upper bound imposed by the absence of timing residuals in measurements of milli-second pulsars 15$]$.

We have shown, therefore, that the inclusion of a cosmological constant can improve the amount of matter power of larger scales, and that an interesting by product of this is a peak in the CMB angular power spectrum. We now illustrate that once the lack of large scale power has been remedied, it is relatively simple improve

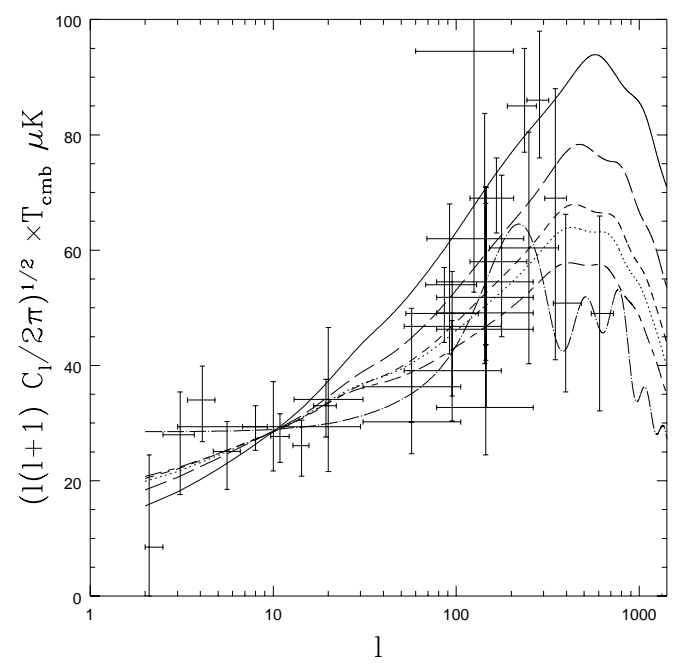

FIG. 4. The CMB power angular power spectrum for the same values of $\Omega_{\Lambda}$ as in Fig. 1. Also included are the observations data points [14 and the standard CDM curve, (dot-dashed) curve for comparison.

the fit to the observations on smaller scales. Ignoring for the moment the constraints on $\Omega_{b} h^{2}$ from Big Bang Nucleosynthesis (BBN), Fig. 5 contains three curves created using the velocity dependent one-scale model and $\Omega_{\Lambda}=0.7$ with (1) $\Omega_{b}=0.05$ and $h=0.7$, (2) $\Omega_{b}=0.15$ and $h=0.7$, (3) $\Omega_{b}=0.125$ and $h=0.5$. Model (1) has the distinction that it fits the amplitude of the small scale matter data $\left(b_{8} \sim 1.0\right)$ though the shape is clearly not right, with a bias of 2.4 on $100 h^{-1} \mathrm{Mpc}$ scales. Models (2) and (3) rectify the small scale slope of the spectrum by increasing the baryon fraction, which damps out small scale power. A similar effect could be obtained by the introduction of hot dark matter.

As one final point we return to the approach adopted in refs. 3, 14 of finding the transition which fits the data best, since the exact nature of the deviation from scaling at the onset of cosmological constant domination has not yet been simulated. We implement a transition using the model described in ref. 四 by varying $\mu$ with $\tau_{T}=10000$, $L_{T}=0.1$ and $\chi=4$, such that the time and length of the transition closely mirror those obtained in the onescale velocity model and the amplitude chosen to give the best fit to the data. The results are shown in Fig. 司 first without bias and, to emphasize the goodness of the fit to the shape of the matter data, with a scale invariant bias of $b=2.0$. This model has been shown by Gawiser and Silk [16] to give a good fit to the entire dataset, including $\mathrm{CMB}$ and matter power spectra, peculiar velocities and cluster abundances.

Our results demonstrate that some of the problems of standard defect scenarios in underproducing power in the large scale matter distribution can be remedied by the introduction of a cosmological constant. Within this scenario, there are two distinct effects, a change in the 


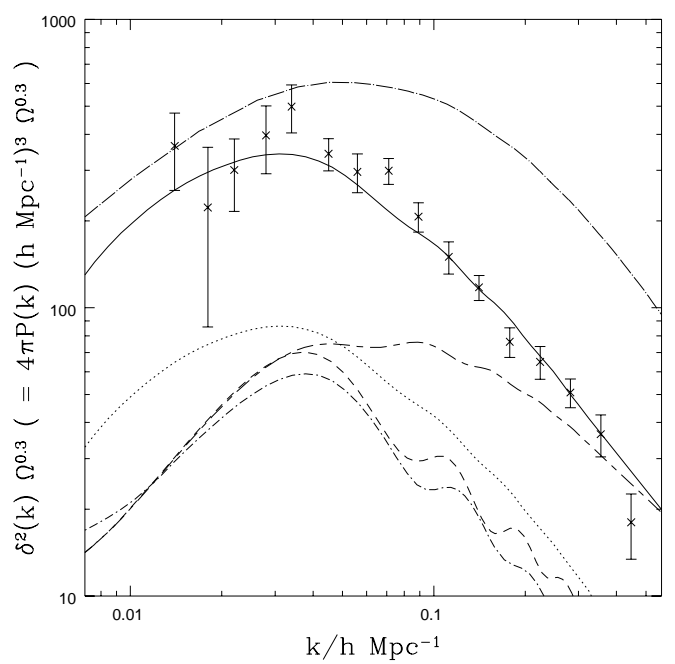

FIG. 5. The matter power spectrum for various models with $\Omega_{\Lambda}=0.7$. Velocity one scale model: (1) $h=0.7$, $\Omega_{b}=0.05$ - long-short dash line; (2) $h=0.7, \Omega_{b}=0.15$ - short dash line; (3) $h=0.5, \Omega_{b}=0.125$ - dot-short dash line. Transition model with $\chi=4$ : unbiased - dotted curve; biased - solid curve.

evolution of the background perturbations, and more importantly a modification to the scaling behaviour of the strings, which both act to allow more matter power on scales of $100 h^{-1} \mathrm{Mpc}$. Using the velocity dependent one scale model we find that biases $b \sim 2$ are required: On first examination, this value still seems unreasonably high [16], but the discrepancy with the data is small enough that these models deserve further consideration. The improvement to the bias does not contradict the findings of refs. [3, 4 ], since it has come about by significant deviation in the scaling behaviour of the strings, due to the presence of the cosmological constant. Our results for the matter power spectrum and bias are in good agreement with ref. [9], lending weight to the simpler techniques employed in that work. It is clear that similar results will also be obtained in an open universe [9.10], and we are currently investigating this case.

Finally, we should comment on our choice of cosmological parameters since this is a subject which is currently evolving rapidly. It appears that the best fit to the power spectrum is attained by using $\Omega_{\Lambda}=0.7$ which is very much in line with the current measurements of cosmological deceleration from supernova type Ia [17] and other independent measurements of the matter density of the universe. We should also note that some of the values of $\Omega_{\mathrm{b}}$ used in Fig. 5 are slightly larger than usually used on the basis of BBN. However, these higher values are not excluded [18] and we only use them here to illustrate that models which have a reasonable $b_{100}$ can be made to fit the observed power spectrum on smaller scales for example, the addition of hot dark matter can have a similar effect. We conclude that the interaction between this model and observations in the coming months will be interesting, and could in the end produce strong support for our model.

We thank U. Seljak and M. Zaldariagga for the use of CMBFAST. We would also like to thank P. Ferreira, N.Turok, E. Gawiser, P. Viana for helpful comments and P. Shellard for enlightening discussions concerning deviations from scaling. This work was supported by PPARC and computations were done at the UK National Cosmology Supercomputing Center, supported by PPARC, HEFCE and Silicon Graphics/Cray Research. RAB is funded by Trinity College.

[1] T.W.B. Kibble, J. Phys. A9, 1387 (1976).

[2] U.L. Pen, U. Seljak and N.Turok, Phys. Rev. Lett. 79, 1615 (1997).

[3] A. Albrecht, R.A. Battye and J. Robinson, Phys. Rev. Lett. 79, 4736 (1997).

[4] A. Albrecht, R.A. Battye and J. Robinson, astro$\mathrm{ph} / 9711121$.

[5] B. Allen, R.R. Caldwell, S. Dodelson, L. Knox, E.P.S. Shellard and A. Stebbins, Phys. Rev. Lett. 79, 2624 (1997).

[6] U. Seljak and M. Zaldarriaga, Ap. J. 469, 437 (1996).

[7] A. Albrecht and A. Stebbins, Phys. Rev. Lett. 68, 2121; ibid 692615 (1992).

[8] P. Ferreira, Phys. Rev. Lett. 74, 3522 (1995).

[9] P.P. Avelino, R.R. Caldwell and C.J.A.P. Martins, Phys. Rev. D56, 4568 (1997).

[10] P.P. Avelino, E.P.S. Shellard, J.H.P. Wu and B. Allen, In prep.

[11] J.A. Peacock and S.J. Dodds, MNRAS 267, 1020 (1994).

[12] C.J.A.P. Martins and E.P.S. Shellard, Phys. Rev. D54, 2535 (1996).

[13] C.J.A.P. Martins and E.P.S. Shellard, Private comm.

[14] The data points have been compiled by M. Tegmark at http://www.sns.ias.edu/ max/cmb/experiments.htm

[15] R.R. Caldwell, R.A. Battye and E.P.S. Shellard, Phys. Rev. D54, 7146 (1997).

[16] E. Gawiser and J. Silk, In preparation.

[17] S. Perlmutter et al, See http://www-supernova.lbl.gov.

[18] K. Olive, astro-ph/9712160. 